\title{
ON THE USE OF ACCELERATED AGING METHODS FOR SCREENING HIGH TEMPERATURE POLYMERIC COMPOSITE MATERIALS ${ }^{1}$
}

\author{
Thomas S. Gates \\ AIAA Associate Fellow \\ Research Scientist, NASA Langley Research Center, Hampton VA 23681 \\ Michael A. Grayson \\ Assistant Director, Center for Biomedical and Bioorganic Mass Spectrometry \\ Washington University, St. Louis, MO 63130
}

\begin{abstract}
A rational approach to the problem of accelerated testing of high temperature polymeric composites is discussed. The methods provided are considered tools useful in the screening of new materials systems for long-term application to extreme environments that include elevated temperature, moisture, oxygen, and mechanical load. The need for reproducible mechanisms, indicator properties, and real-time data are outlined as well as the methodologies for specific aging mechanisms.
\end{abstract}

\section{BACKGROUND}

In 1996, at the request of NASA, the National Research Council's National Materials Advisory Board (NMAB) published a report (Starke [1]) on accelerated aging of future aircraft related materials. The NMAB study committee was established to (1) provide an overview of long-term exposure effects; (2) recommend improvements to analytical methods and approaches to accelerate laboratory testing; and (3) identify research needed to develop and verify the required testing, predictive analytical capabilities, and evaluation criteria. The NMAB report identified issues related to the aging of aluminum, titanium, polymer matrix composites, and ceramic matrix composites. Suggestions were outlined for accelerated evaluation approaches and analytical methods to characterize the durability of future aircraft materials and structures throughout their service life. The current work specifically addresses issues in accelerated aging of polymer matrix composite materials suitable for high temperature applications.

\section{INTRODUCTION}

The long-term exposure of aerospace, polymeric composite materials to the use-environment will eventually result in change(s) in the original properties of the material. This process is loosely referred to as "aging". This material aging may translate to structural changes in mission-critical components which for an aerospace platform can have a potentially catastrophic effect on both the vehicle and its payload. Therefore, studying and understanding the aging process in high performance aerospace materials is critical to their proper design, construction and safe operation.

Verified accelerated aging methods are needed to provide guidance for materials selection and to accurately assess aging of new materials. The concept of accelerated aging can be interpreted many different ways and it is therefore important that a common definition of some important terms be established before going into further detail. Three terms are of particular importance: environmental stress factor,

\footnotetext{
1 "Copyright 1998 by the American Institute of Aeronautics and Astronautics, Inc. No copyright is asserted in the United States under Title 17, U.S. Code. The U.S. Government has a royalty-free license to exercise all rights under the copyright claimed herein for Governmental purposes. All other rights are reserved by the copyright owner."
} 
critical degradation mode, and accelerated aging. Environmental stress factor is the general term for specific use-environment conditions; i.e. heat, moisture, mechanical load, etc. are all environmental stress factors. Critical degradation mode or mechanism refers to the fact that all polymer systems are more susceptible to attack by a specific set of environmental stress factors. The degradation mechanism that results in a significant loss in any important bulk physical property of the material system when exposed to environmental stress factors inside the limits of the use-environment is the critical degradation mechanism. Accelerated aging is defined as the process or processes required to accelerate a specific mechanism or mechanisms relative to a baseline aging condition; thereby resulting in the material reaching the same aged end-state as a realtime aged material, but in less time.

Only by understanding how each aging mechanism affects a given material system can it be determined if that aging mechanism can be properly accelerated. In the simplest case, aging is associated with a single mechanism, in which case acceleration of this mechanism will allow meaningful accelerated aging methods to be developed. More likely, the aging process involves several different mechanisms that may or may not act synergistically, complicating the problem significantly. Irrespective of these difficulties, it is critical that the mechanisms underlying aging in high performance material systems be studied and explored. Without an understanding of these underlying processes, there is little hope that accelerated aging studies will be of much use in the materials science community.

\section{OBJECTIVE}

The highly empirical approaches taken for the majority of accelerated aging studies dictates that the primary objective of an accelerated aging method is to screen and characterize new material systems. Material testing is a costly process that often involves many materials-related disciplines and a wide variety of laboratory equipment. It is recognized that while longterm, real-time testing is required to fully assess the durability of materials, accelerated aging may reduce the expense and time involved by significantly narrowing the field of acceptable candidate materials which would go into long-term qualification tests. In addition to materials screening, accelerated aging may help determine residual service life of existing structures and suggest directions for product improvements. This type of information may then lead to changes in the standard practice and provide quantitative rationale for manufacturers and fabricators to follow new and improved specific procedures.

The empirical methods for accelerated aging may address the concerns for specific applications and environments, but the need for predicting performance in broader service conditions will require the development of empirical methods coupled with analytical methods. The development of accelerated aging methods requires extensive testing to define critical environmental stress factors and their interactions. This testing provides insight into how materials behave and input for the development of analysis methods to predict material performance under various conditions of load, temperature, and environment. A comparison of mechanical properties, damage modes, and physical parameters; such as weight loss, changes in glass transition or fracture toughness; from accelerated testing with those from real time testing serves to validate accelerated aging methods.

\section{REQUIREMENTS FOR ACCELERATED}

\section{TESTING}

While the details of the aging process in polymeric composites can be quite complicated in a given material system, it is worth pausing to consider that the general process of aging is fairly straightforward. Whether or not we understand the relationship between the molecular and morphological structures of a polymer system and its bulk mechanical properties, the fact remains that those bulk properties are determined by features at the molecular and microscopic level. During aging, one or more environmental stress factors act on the bulk polymer. The effects of these stress factors are translated down to the microscopic/molecular level where some change occurs in the polymer network or chain via some mechanism. This modification of structure is translated back to the macroscopic level and observed as a change in bulk mechanical performance. During real-time aging, this continual evolution of bulk properties is slow and frequently unnoticeable. It is the challenge of an accelerated aging program to speed up the effects of exposure to environmental stress factors without departing from the underlying mechanisms that give rise to molecular level changes.

\section{Reproducible Mechanisms}

We employ this simple generalized understanding of the aging process in our development here. The most important requirement to keep in mind during the development of an accelerated aging method is that it

2

American Institute of Aeronautics and Astronautics 
must replicate those changes that occur in the real world. Thus emphasis must be put on the need to understand and reproduce degradation mechanisms associated with each accelerated aging condition. A mechanistic approach to this requirement would require complete knowledge of all relevant degradation mechanisms and the recognition that competing mechanisms may proceed at different rates as well as interact synergistically. Ideally, aging conditions can be chosen to accelerate material aging to a sufficient degree that one can establish rate information.

Indicator Properties

Initially, accelerated aging is based on results from exploratory tests from which predictive models provide a means for selecting aging parameters outside the intended use-environment. These aging conditions are chosen in order to reduce the time required to achieve a desired material end-state, but without changing the damage mechanisms identified for the operational environment. Beyond these exploratory tests, additional studies must be conducted to identify material properties that are easy to measure and that can be reliably correlated to changes in mechanical properties. These material "indicator" properties form the basis for development of more economical accelerated aging schemes for screening new materials and for evaluating the status of materials in long-term aging.

\section{Real-Time Data}

Another significant requirement for accelerated aging studies is the coupling of mechanical property data from accelerated aging of materials with data from real-time aging. The comparison with real-time data will determine the accelerating factor for any given degradation mechanism. This correlation with results from real-time exposure dictates the need to monitor all environmental stress factors and determine sensitivity of the factors to variations in parameters (e.g., temperature, humidity).

For most commercial, polymeric composite material systems, the cost of developing an extensive real-time database will force laboratories to collaborate and develop national databases. This type of coordinated effort will rely on the use of standardized test methods and data reporting. All reported data should indicate potential long-term behaviors by acceleration of appropriate mechanisms and should include acceptance test data. Environmental stress factors such as temperature, load, moisture exposure, etc. can have an effect on degradation mechanisms. Normally, one or two environmental stress factors dominate the aging of a material system. Each aging mechanism should be investigated separately in order to determine the dominant environmental stress factors. This provides a series of tests that can be performed to determine the critical degradation mechanism of a given material system. As an example of this type of test matrix, table 1 shows the types of tests run under the NASA High Speed Research [2] Program, Materials Durability Task. As indicated by table 1, a number of composite material systems were tested under real time conditions across a range of exposure or aging conditions. Data from this program formed the basis for validation of accelerated testing.

\section{METHODOLOGIES FOR POLYMER}

\section{MATRIX COMPOSITES}

The three basic constituents of advanced polymer matrix composites (PMC's) are fiber, interface, and matrix. In many circumstances the polymeric matrix can be the key constituent that contributes to degradation or changes in durability of PMC's. Changes in composite stiffness, strength and fatigue life can all be related to changes in the mechanical properties of the polymer matrix. The accelerated aging philosophy developed by several research programs and recently summarized by Bank, et al. [3] has promoted individual test methods combined in an integrated scheme to provide an accurate method for understanding the different contributions of various degradation mechanisms to durability.

Classes of Degradation Mechanisms

Degradation (aging) mechanisms for PMC's are divided into three classes: chemical, physical, and mechanical. These three mechanisms may be additive or subtractive depending on the environmental and mechanical loads.

\section{Chemical Aging}

Chemical aging refers to an irreversible change in the polymer chain/network through mechanisms such as cross-linking or chain scission. Chemical degradation mechanisms include thermo-oxidative, thermal, and hydrolytic aging. At typical operating temperatures $\left(75^{\circ} \mathrm{F}-350^{\circ} \mathrm{F}\right)$, cross-linking and oxidation are the dominant chemical aging mechanisms. Thermo-oxidative degradation becomes increasingly important as the exposure temperature and time increase. Frequently, such aging results in an increase in cross-linking density that can severely affect the mechanical properties by densification and increasing the glass transition temperature $T_{\mathrm{g}}$.

\section{Physical Aging}

Physical aging will occur when a polymer is cooled below its $\mathrm{T}_{\mathrm{g}}$, and the material evolves toward thermodynamic equilibrium. This evolution is characterized by changes in the free volume, enthalpy, and entropy of the polymer and will produce 
measurable changes in the mechanical properties (Struik [4], McKenna [5]). Physical aging is thermoreversible by heating the polymer above its $T_{g}$ and subsequently quenching the material. Temperature and thermal history have a strong influence on the rate of physical aging.

Mechanical Degradation
Mechanical degradation mechanisms are
irreversible processes that are observable on the macroscopic scale. These degradation mechanisms include matrix cracking, delamination, interface degradation, fiber breaks, and inelastic deformation; and thus have a direct effect on engineering properties such as bulk stiffness and strength. In some cases, mechanical degradation mechanisms dominate only after chemical degradation mechanisms have modified the polymer properties in such a way that loads and stresses are not tolerated in the aged materials as they would be in the unaged material.

\section{Real-time Testing}

The methodology for real-time testing of a durable composite over the service life of typical applications has been detailed in several standards documents such as provided by MIL-HNBK-17 and ASTM. These standards provide guidance on the selection of methods to assess degradation due to a variety of mechanical factors. When considering the effects of environmental stress factors on durability, these standard test methods form a starting point for the development of new accelerated test methods.

The assessment of environmental stress factors in the aging of polymer matrix composite materials is crucial to the development of aging scenarios capable of accelerating the degradation process. This assessment must include tasks which concentrate on long term aging, determination of acceleration factors, and analysis development. Essentially, the long-term aging task provides the baseline data against which all accelerated work must be compared. Additional aging is performed investigating the individual and synergistic effects of specific environmental stress factors on the degradation process. For example, environmental stress factors under consideration may include aging at temperatures greater than the usetemperature but less than the glass transition temperature, aging in different gaseous environments, aging under load, exposure to ultra-violet radiation, and exposure to moisture. Data from such aging is used to determine which environmental stress factors dominate the degradation process and which result in the same degradation modes as observed in the realtime, long-term aging study. Once an understanding of the material behavior is obtained models can be developed to predict material performance under different exposure and loading conditions. These models can be used to simulate the effects of different aging profiles to help formulate an accelerated aging scenario.

\section{GENERAL PROCEDURES}

At the beginning of an accelerated aging program for PMC's, attention should be focused on the determination of critical degradation mechanism(s). The key to this task is to determine the material performance after systematic exposure to one or more environmental stress factors. Material performance is defined by a set of indicators that measure a specific property for the material. The indicators will be different dependent upon the mechanism of interest. The suggested procedure is as follows:

a) Identify material by class (i.e. thermoplastic, thermoset).

b) Identify mechanism to evaluate (i.e. thermal stability, matrix cracking, etc.).

c) Choose an environmental stress factor for aging.

d) Conduct aging experiment within limits of the chosen environmental stress factor using established methods.

e) Perform post-aging tests with indicators sensitive to changes in material performance and compare results to unaged values.

The results of such a study will allow one to evaluate whether this particular degradation mechanism will be critical for the given application. This procedure can be repeated for all degradation mechanisms of interest for that material. It should not introduce extraneous damage/degradation mechanisms nor should it omit any known degradation mechanisms. Additionally, the set of mechanical properties or indicators chosen for screening should be:

- Those most critical from the structural performance viewpoint

- Those most sensitive to degradation.

Identify Material Type

The first step mentioned above requires material identification. For this purpose, a thermoset is defined as a cross-linked polymer network that hardens to final shape after cool down from the forming temperature and is incapable of being reshaped. A thermoplastic material is a linear polymer of amorphous, semicrystalline, or mixed morphology. A thermoplastic softens on heating to a state where the shape may be changed by physical forces and resolidifies on cooling. In principle, the process of softening and solidifying may be repeated indefinitely.

A great deal of work has been performed using these principles over the last five years. While it is not 
possible to detail the results of all of this work, it is possible to report here some generalized conclusions. Assess Aging by Thermo-Oxidative Degradation

In the polyimide material systems, the oxidation reaction occurs through a radical chain process (David and Sims [6], Schnabel [7], White and Turnbull [8]) and is referred to as autoxidation. The effects of oxygen exposure during aging can only be determined if compared against aging in an inert environment. For example, as shown in figure 1, samples were aged at temperatures above the upper use-environment temperature, but below the glass transition temperature in both the presence and absence of oxygen. Some indicators that monitor thermo-oxidative degradation are:

1. Weight: Initial weight loss of a polymer exposed to thermo-oxidative environment may occur due to loss of moisture and residual volatiles and is not related to polymer breakdown. Usually, after several hundred hours of exposure, the sample weight stabilizes and any additional weight loss is indicative of thermo-oxidative degradation. This is shown in figure 1 by comparing weight loss for three different exposure environments. Once polymer degradation occurs, the evolution of gaseous degradation products accompanies weight loss.

2. Physical Changes: Optical measurements, such as changes in color, surface texture, and crack density are indicators of thermo-oxidative degradation

3. Glass Transition Temperature: Changes in the glass transition temperature are frequently observed by dynamic mechanical analysis. Usually, an increase in $\mathrm{Tg}$ suggests chain extension or network crosslinking. Figure 1 shows an example how the Tg can increase due to long term exposure. A decrease is normally associated with chain scission.

4. Mechanical Properties: Most residual properties such as tension strength, compression strength, and stiffness do not make good indicators of thermo-oxidative degradation early in the aging process. However, more subtle mechanical properties such as fracture toughness are sensitive indicators of short-term aging.

Assess Aging by Thermal Degradation

Elevated temperature aging in polymers in the absence of oxygen can also lead to changes in material properties due to additional cross-linking and/or chain scission. Analytical models capable of predicting the long-term changes associated with chemical aging are rare. A recent model, which correlates change in mechanical properties due to additional cross-linking, is given in Zhou [9]. Generally, thermoset systems initially tend to embrittle during purely thermal aging. Depending upon thermoset chemistry, these systems may either continue to embrittle or start to degrade significantly. Indicators that are useful for tracking thermal degradation are much the same as for thermooxidative degradation. As an example, figure 2 shows a comparison between properties measured for room temperature and elevated temperature fatigue of a graphite/bismaleimide composite (Johnston and Gates [10]). As shown, the changes incurred during the elevated temperature fatigue (case B) included loss in weight, increase in glass transition temperature, and increase in crack density.

1. Weight: Initial weight loss of a polymer exposed to a purely thermal environment may occur due to loss of moisture and residual volatiles, just as in thermo-oxidative degradation. However, the magnitude of weight loss for equal exposure times and temperatures is generally much less in purely thermal degradation than it is in thermo-oxidative degradation.

2. Physical Changes: Optical measurements, such as changes in color, surface texture, and crack density are indicators of thermal degradation. Frequently, these changes are less noticeable in thermally aged samples compared to those aged under the same conditions with oxygen present.

3. Glass Transition Temperature: Changes in the glass transition temperature are frequently observed by dynamic mechanical analysis. These changes are considerably smaller for equivalent aging conditions than for samples aged in the presence of oxygen.

4. Mechanical Properties: Most residual properties such as tension, compression strength, and stiffness do not make good indicators of thermal degradation early in the aging process. However, more subtle mechanical properties such as fracture toughness are sensitive indicators of short-term aging.

Assess Aging by Hydrolytic Degradation

The degree of plasticity in a polymer will increase due to long-term exposure to moisture. Moisture exposure has two important components: 1) diffusion of water into the sample and 2) plasticization of the matrix. Depending upon the diffusion coefficient of water in the polymer system, significant differences in water concentration can exist from one region of the sample to the next, imposing additional stresses on the system. Some systems have also demonstrated a decrease in microcracking fracture toughness when simultaneously exposed to water and mechanical stress. At exposure conditions well inside the useenvironment; temperature $175 \mathrm{~F}$, humidity $50 \%$, and tensile stress equal to residual internal stresses in a quasi-isotropic laminate, microcracks will develop in less than 5000 hours of exposure in some polymer systems. Note that all of the environmental stress factors are well within the limits of the intended use- 
environment, yet significant degradation of the polymer matrix is observed. Such microcracking will subsequently affect the rates of moisture absorption/desorption during repeated hygro-thermal cycling.

An example of this is provided in figure 3, which shows the moisture absorption, desorption curves for a graphite thermoplastic polyimide. This is a perfect example of a critical degradation mode. The presence of internal stresses formed microcraks in the laminate which subsequently allowed for new pathways for moisture uptake. In this case, the environmental stress factors are heat, moisture, and mechanical load. Indicators that are useful for tracking hygrothermal stability are:

1. Physical Changes: An increase in crack density may be observed after exposure. As shown in figure 3 , anomalous weight change behavior may be noted during cyclic exposure with the time to saturation and drying shortened by orders of magnitude following microcrack formation.

2. Mechanical Properties: Fracture toughness is particularly sensitive to hygro-thermal degradation. Other engineering properties such as residual tension and compression strength, and stiffness, are also affected to a lesser extent.

Assess Physical Aging

As noted earlier, physical aging involves subtle changes in thermodynamic properties of the polymer matrix. A measure of the rate and hence acceleration of physical aging using isothermal creep tests has been performed on a number of polymer systems with low glass transitions (McKenna [11], Crissman [12], Sullivan [13], Read [14], Read [15], Janas [16]). Physical aging of polymers and polymer composites usually employs a method that relies on short-term tests and the concept of an aging-induced shift factor. This test method provides an approach to obtain quantitative relationships among the short-term, timedependent experimental data, aging-free creep master curves, and the long term behavior. For PMC's, details of these test procedures are provided in Veazie and Gates [17], Gates and Feldman [18]. All of the experimental and analytical procedures described in these documents make use of the principles of timetemperature superposition (TTSP) and time-aging time (TASP) superposition. The fundamental assumption in both cases is that the time-based response can be accelerated. For the case of TTSP, an increase in temperature will accelerate the time-dependent response (e.g. creep, relaxation) however changes in the aging behavior are not accounted for. To accelerate the aging effects, both TTSP and TASP must be utilized. The TTSP and TASP shift factors and the associated material master curves are found through test. These parameters then form the basis of the material related inputs to the predictive models.

Models for prediction of long-term viscoelastic behavior that include the effects of physical aging are available in the literature (Peng [19], Dean [20], Brinson and Gates [21]). Most of these models use an integral expression where the effect of physical aging is normally accounted for by modifying the expression for the viscoelastic compliance. The model proposed by Brinson and Gates and expanded by Bradshaw and Brinson [22] was successfully employed to model the response of a graphite/thermoplastic laminate loaded both in tension and compression over a range of subTg temperatures Gates, et al. [23]. The indicator used to characterize the physical aging of polymer composites is as follows:

1. Mechanical Properties: Time-dependent tests, which can be used to assess the degree of physical aging, include creep and stress relaxation. These tests should be made so that the material remains within the linear viscoelastic range. Static residual properties, which indicate a change in behavior due to physical aging, include tension and compression strength and stiffness of notched and unnotched specimens.

For the physical aging mechanism, Struik [4] suggested that loading a material to a nonlinear stress level will apparently "deage" it. The explanation proposed by Struik is that large deformation processes caused by high stress, generate free volume and rejuvenate the sample, partially erasing the previous aging. An alternative explanation for the reduction of shift rate with high stresses has been proposed by Lee [24]. They stated that an increase in the amplitude of the stress applied in the physical aging experiment results in a decrease in the shift rate simply because the changes in structure accompanying volume recovery affect the nonlinear response differently at large stresses than at small ones. Investigations on the effects of stress during aging of composites was performed by Gates and Feldman [25].

Assess Aging by Mechanical Degradation

Mechanical degradation mechanisms are those associated with the initiation of damage or the growth of pre-existing damage due to the application of stress or deformation. Often these mechanisms are the result of prior physical or chemical aging which changes the constituent mechanical properties. They also are aided by other factors such as initial defects or flaws in the materials. The dominant mechanical mechanisms include matrix cracking, delamination, fiber breaks, and inelastic deformation. 
Matrix Cracking: Given a sufficient mechanical load, matrix cracking will occur in PMC's. Cracks that initiate due to localized stress concentrations typically act as a stress relieving mechanism. These cracks have the same effect as lowering the stress concentration factor and allowing more load to be carried by the laminate and, therefore, increase the gross strength. Conversely, matrix micro-cracks that initiate in offaxis plies result in a loss of laminate stiffness. The magnitude of the load and the extent of the environmental stress factors determine the degree of matrix micro-cracks. An example of the effects of load and temperature are shown in figure 4 (Johnston and Gates [10]). In this figure, the micro-crack density data from the composite laminate shows that at all temperatures, crack density will increase with an increase in the mechanical load and that even in the absence of mechanical load, high temperature exposure alone may be sufficient to generate substantial microcracks.

In general, micro-crack densities increase as the exposure time increases and additional long-term exposure increases the probability of delamination and eventually fiber breakage. Preferred methods of accelerating this mechanism are increasing the rate and magnitude at which the mechanical and thermal spectra are applied. The indicators used to characterize the microcracking of polymer composites are as follows:

1. Physical Changes: An increase in crack density may be observed after static or fatigue loading.

2. Mechanical Properties: Static residual properties, which indicate a change in stiffness, include tension and compression of notched and unnotched specimens.

Delamination: Polymer composite laminates delaminate when through-the-thickness stresses exceed out-of-plane strength properties (O'Brien [26], O'Brien [27], Adams [28], Hooper [29]). This type of damage mechanism can significantly reduce the compressive strength of the laminate by reducing the stability in the region where the delamination occurs thereby increasing the likelihood of a buckling failure mode. The level of applied stress directly influences the incidence and rate of delamination. Preferred methods of accelerating this mechanism are by increasing the rate at which the mechanical and thermal spectra are applied. The indicator used to characterize the delamination of polymer composites is as follows:

1. Physical Changes: Void formation detected by non-destructive evaluation of laminate integrity using ultrasonic C-scans.

Fiber Breakage: Fiber failure in PMC's occurs when mechanical loads exceed fiber tensile strength or from shear-induced failure during transverse impact. Increases in environmental stress factors such as temperature or moisture may decrease fiber strength as well as modify the matrix response to externally applied loads. Preferred methods of accelerating this mechanism are by increasing the rate at which mechanical fatigue loads and thermal cycles are applied. The indicators used to characterize the fiber failure of polymer composites are as follows:

1. Mechanical Properties: Static residual properties, which indicate a change in stiffness and strength, include tension and compression of notched and unnotched specimens.

Inelastic Deformation: This type of mechanical damage can occur in a polymer matrix composite due to plasticity, creep and stress relaxation. Each of these topics is defined below. However, the dominant affect on a laminate would be observed as a change in its matrix dominated properties such as shear and transverse stiffness. This mechanism can be accelerated by exposure to higher stresses and higher temperatures.

Plasticity: If the stress in a material is too high its response is no longer elastic. The limiting stress above which this occurs is called the elastic limit. The strain that remains after removal of the stress is called the inelastic strain. In some materials, the strain continues to increase for a short while after the load is fully applied, and then remains constant under a fixed load, but a permanent strain remains after the stress is removed. This permanent strain is called the plastic strain. Plastic strain is defined as time independent although some time dependent strain is often observed to accompany plastic strain.

Creep: Creep is the slow continuous deformation of a material under constant stress. For small strains, constant load and constant stress experiments are the same. The first stage in which creep occurs at a decreasing rate is called primary creep; the second, called secondary stage, proceeds at a nearly constant rate; and the third or tertiary stage occurs at an increasing rate and terminates at fracture.

Stress Relaxation: Stress relaxation is the gradual decrease in stress of a material subjected to a constant strain. This stress may asymptotically reach a limiting value slowly over time.

\section{SUMMARY AND CONCLUDING REMARKS}

Historically, the two dominant means for accelerating aging in PMC's are the use of increased levels of mechanical stress and temperature. Increasing mechanical stress has several effects at the molecular 
level: diffusion of gases and liquids into the polymer matrix is enhanced and some population of chemical bonds in the polymer chain will be loaded to a higher level, thereby reducing the energy needed to rupture the bond. Increasing the temperature accelerates all thermally activated rate processes. It will also reduce the activation energy of chemical bond rupture in the polymer. Elevated temperature (sub-Tg) will also increase the free volume in the polymer, hence decreasing the time needed to age to thermodynamic equilibrium. Increased temperature is also usually associated with decreases in both strength and stiffness in PMC's and will lead to increased ductility and strain to failure. Unfortunately, the use of elevated temperature for acceleration of the aging mechanism(s) may promote degradation mechanisms that do not occur at use-environment temperatures or alter the rates so that degradation may not be accelerated proportionally.

For chemical aging mechanisms, stress, or mechanical load, may increase the probability of bond rupture within the polymer. Residual stress or the externally applied stress on the chemical bond can accelerate chain scission caused by chemical reaction. It has also been found that stress can alter the effective activation energy for a chemical reaction. The use of stress to accelerate physical aging is not recommended due to a lack of clear understanding of the effect of applied stress on physical aging. Increased stress has been traditionally used as the primary means for accelerating mechanical degradation. The occurrence of microcracks, fiber breaks, and delaminations will all be accelerated through the application of increased static or fatigue stress.

Aside from temperature and stress, secondary accelerators such as moisture, partial pressure of oxygen, geometry, and layup should be considered for PMC's. The application of a particular accelerator or set of accelerators will be dictated by the dominant degradation mechanism for each use-environment. One must also consider that aging performed in the standard environment may actually represent an accelerated aging case for material systems that don't operate in the standard environment. As an example, consider real-time isothermal aging used to establish baseline conditions. For this example, the level of oxygen concentration in laboratory air in aging ovens exceeds by several orders of magnitude the concentration of oxygen that a laminate on a supersonic aircraft would see when it is at altitude undergoing operational load. Furthermore, one should take into account the fact that some environmental stress factors will play an insignificant role in the aging of a material because they are simply not reproduced in the use-environment. For example, when moisture uptake occurs the temperature will be low. Therefore, although moisture is present, it will diffuse very slowly into the laminate matrix. During ascent and operation of high performance aircraft, most moisture absorbed by the laminate will be desorbed quite rapidly due to the increase in the temperature at operational conditions. Thus, the equilibrium water content of the in-service laminate will never approach that of the lab specimen, even over the lifetime of the aircraft.

Prior to the development of accelerated test methods, several limiting factors should be recognized. Perhaps the most important limiting factor is that the "short term test" can actually take several years to develop and validate. Hence, time available for methods development may control the final outcome of the test program. Other limiting factors to consider include:

- Lack of in-situ NDE methods.

- Some property behavior cannot be accelerated.

- Scaling or size effects may play a role.

- Synergistic mechanisms can confound data analysis.

- Lack of integration of field data and lab data.

- Site specific environmental factors must be addressed.

- Need to mitigate risk may stifle innovation.

- Introduction of new materials may make some data obsolete.

- Lack of standards for data collection.

Clearly, the study of aging, accelerated aging, and the effects of aging on high performance laminated materials is a challenging and difficult task. Nevertheless, it is imperative that a scientific knowledge base is built up as the demands on material systems increase on all fronts. Only through longterm, dedicated perseverance in addressing the difficult and complex issues surrounding this area of research can we expect to extend the use of modern materials systems safely and reliably to the most demanding, challenging and rewarding applications.

\section{REFERENCES}

[1] Starke, E. A. J., "Accelerated Aging of Materials and Structures, The Effects of Long-Term ElevatedTemperature Exposure," National Materials Advisory Board, National Research Council, Washington, D.C. NMAB-479, 1996.

[2] National Research Council, "U.S. Supersonic Commercial Aircraft," National Academy Press, Washington, D.C. 1997. 
[3] Bank, L. C., Gentry, T. R., and Barkatt, A., "Accelerated Test Methods to Determine the LongTerm Behavior of FRP Composite Structures: Environmental Effects," Journal of Reinforced Plastics and Composites, Vol. 14, pp. 559-587, June, 1995.

[4] Struik, L. C. E., Physical Aging in Amorphous Polymers and Other Materials. New York: Elsevier Scientific Publishing Company, 1978.

[5] McKenna, G. B., "On the Physics Required for the Prediction of Long Term Performance of Polymers and Their Composites," Journal of Research of the National Institute of Standards and Technology, Vol. 99, pp. 169-189, 1994.

[6] David, A. and Sims, D., Weathering of Polymers. London: Applied Science Publishers, 1983.

[7] Schnabel, W., Polymer Degradation: Principles and Practical Applications. New York: Hanser International, 1981.

[8] White, J. R. and Turnbull, A., "Review Weathering of Polymers: Mechanisms of Degradation and Stabilization, Testing Strategies and Modeling," Journal of Materials Science, pp. 584-613, 1994.

[9] Zhou, J., "A Constitiutive Model of Polymer Materials Including Chemical Aging and Mechanical Damage and its Experimental Verification," Polymer, Vol. 34, pp. 4252-4256, 1993.

[10] Johnston, W. M. and Gates, T. S., "The Effects of Stress and Temperature on the Open-Hole Tension Fatigue Behavioru of a Graphite/Bismaleimide Composite," Composite Materials: Fatigue and Fracture, Seventh Volume, ASTM STP 1330, R. B. Bucinell, Ed. Philidelphia: American Society for Testing and Materials, pp. 179-198, 1998.

[11] McKenna, G. B., "Aging of Polymeric Resins: Implications for Composite Performance," presented at Proceedings of the Conference of the American Chemical Society, 1992, April.

[12] Crissman, J. M., McKenna, G. B., "Physical and Chemical Aging in PMMA and Their Effects on Creep and Creep Rupture Behavior," Journal of Polymer Science: Part B: Polymer Physics, Vol. 28, pp. 14631473, 1990.

[13] Sullivan, J. L., Blais, E. J., and Houston, D, "Physical Aging in the Creep Behavior of Thermosetting and Thermoplastic Composites," Composites Science and Technology, Vol. 47, pp. 389403, 1993.

[14] Read, B. E., Dean, G. D., Tomlins, P. E., "Effects of Physical Aging on Creep in Polypropylene," Polymer, Vol. 29, December, 1988.

[15] Read, B. E., "Analysis of Creep and Physical Aging in Glassy Polymers," Journal of NonCrystalline Solids, Vol. 131-133, pp. 408-419, 1991.
[16] Janas, V. F., McCullough, R. L., "The Effects of Physical Aging on the Viscoelastic Behavior of a Thermoset Polyester," Composite Science and Technology, Vol. 30, pp. 99-118, 1987.

[17] Veazie, D. R. and Gates, T. S., "Compressive Creep of IM7/K3B Composites and the Effects of Physical Aging on Viscoelastic Behavior," Experimental Mechanics, Vol. 37, pp. 62-68, 1997.

[18] Gates, T. S. and Feldman, M., "Effects of Physical Aging at Elevated Temperatures on the Viscoelastic Creep of IM7/K3B," Composite Materials: Testing and Design (Twelfth Volume), ASTM STP 1274, R. B. Deo and C. R. Saff, Eds. Philadelphia: American Society for Testing and Materials, pp. 7-36, 1996.

[19] Peng, S. T. J., "Constitutive Equations of Aging Polymeric Materials," Journal of Materials Science, Vol. 20, pp. 1920-1928, 1985.

[20] Dean, G. D., Read, B. E., and Tomlins, P. E., “A Model for Long-Term Creep and the Effects of Physical Aging in Poly(butylene Terephthalate," Plastics and Rubber Processing and Applications, Vol. 13, pp. 37-46, 1990.

[21] Brinson, L. C. and Gates, T. S., "Effects of Physical Aging on Long Term Creep of Polymers and Polymer Matrix Composites," International Journal of Solids and Structures, Vol. 32, pp. 827-846, 1995.

[22] Bradshaw, R. D. and Brinson, L. C., "Physical Aging in Polymers and Polymer Composites: An Analysis and Method for Time-Aging Time Superposition," Polymer Engineering and Science, Vol. 37, pp. 31-44, 1997.

[23] Gates, T. S., Veazie, D. R., and Brinson, L. C., "Creep and Physical Aging in a Polymeric Composite: Comparison of Tension and Compression," Journal of Composite Materials, Vol. 31, pp. 2478-2505, 1997.

[24] Lee, A., McKenna, G. B., "The Physical Aging Response of an Epoxy Glass Subjected to Large Stresses," Polymer, Vol. 31, pp. 423-430, 1990.

[25] Gates, T. S. and Feldman, M., "Time Dependent Behavior of a Graphite/Thermoplastic Composite and the Effects of Stress and Physical Aging," NASA Langley Research Center, NASA Technical Memorandum 109047, November 1993.

[26] O'Brien, T. K., "Mixed-Mode Strain Energy Release Rate Effects on Edge Delamination of Composites," Effects of Defects in Composite Materials, ASTM STP 386. Philadelphia: American Society for Testing and Materials 1984.

[27] O'Brien, T. K., "Analysis of Local Delaminations and Their Influence on Composite Laminate Behavior," Delamination and Debonding of Materials, ASTM STP 876, W. S. Johnson, Ed. 
Philadelphia: American Society for Testing and Material 1985.

[28] Adams, D. F., Zimmerman, R. S., and Odom, E. M., "Frequency and Load Ratio Effects on Critical Strain Energy Release Rate Gc Thresholds of Graphite/Epoxy Composites," Toughened Composites, ASTM STP 937, N. J. Johnston, Ed. Philadelphia: American Society for Testing and Materials 1987.

[29] Hooper, S. J., Toubia, R. F., and Subramanian, R., "Effects of Mositure Absorption on Edge Delamination, Part I: Analysis of the Effects of Nonuniform Moisture Distributions on Strain Energy Release Rate," Composite Materials: Fatigue and Fracture (Third Volume), ASTM STP 1110, T. K. O'Brien, Ed. Philadelphia: American Society for Testing and Materials 1991. 


\begin{tabular}{|c|c|c|c|c|c|c|}
\hline & & \multicolumn{5}{|c|}{ Long-Term Aging Condition } \\
\hline \multicolumn{2}{|c|}{ Material } & Isothermal & Isothermal & Thermally & Isothermal & Thermo- \\
\hline Thermoplastic & IM7/PIXA & $\bar{X}$ & $\bar{X}$ & $\mathbf{X}$ & $\overline{\mathbf{X}}$ & \\
\hline Polyimide & IM7/K3B & $\bar{X}$ & $\bar{X}$ & $\overline{\mathbf{X}}$ & $\overline{\mathbf{X}}$ & $\bar{X}$ \\
\hline Polyimide & IM7/PETI-5 & $\bar{x}$ & $\bar{x}$ & $\bar{X}$ & $\bar{x}$ & $\bar{x}$ \\
\hline BMI & IM7/5260 & $\bar{x}$ & $\bar{x}$ & $\bar{x}$ & $\bar{X}$ & $\bar{x}$ \\
\hline BMI & IM7/5250-4 & $\overline{\mathbf{X}}$ & $\bar{X}$ & $\mathbf{X}$ & $\mathbf{X}$ & $\bar{X}$ \\
\hline
\end{tabular}

Table 1. Test matrix for long-term aging of polymeric composites performed under the NASA High Speed Research, Materials Durability program.
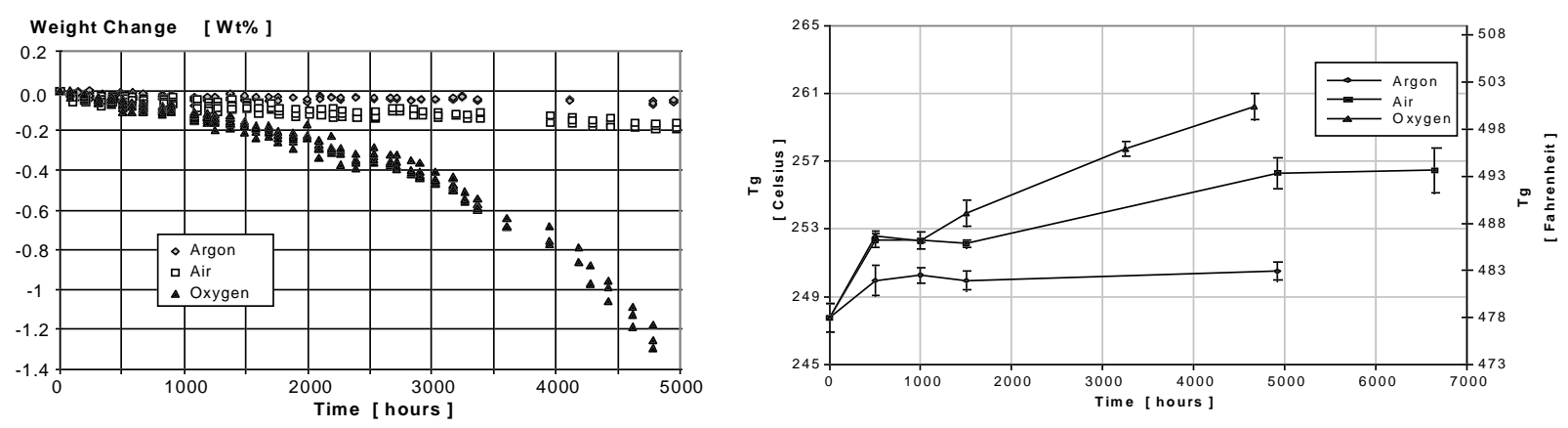

Figure 1. Effects of oxygen content on the measured change in weight and glass transition temperature of a graphite/thermoplastic.
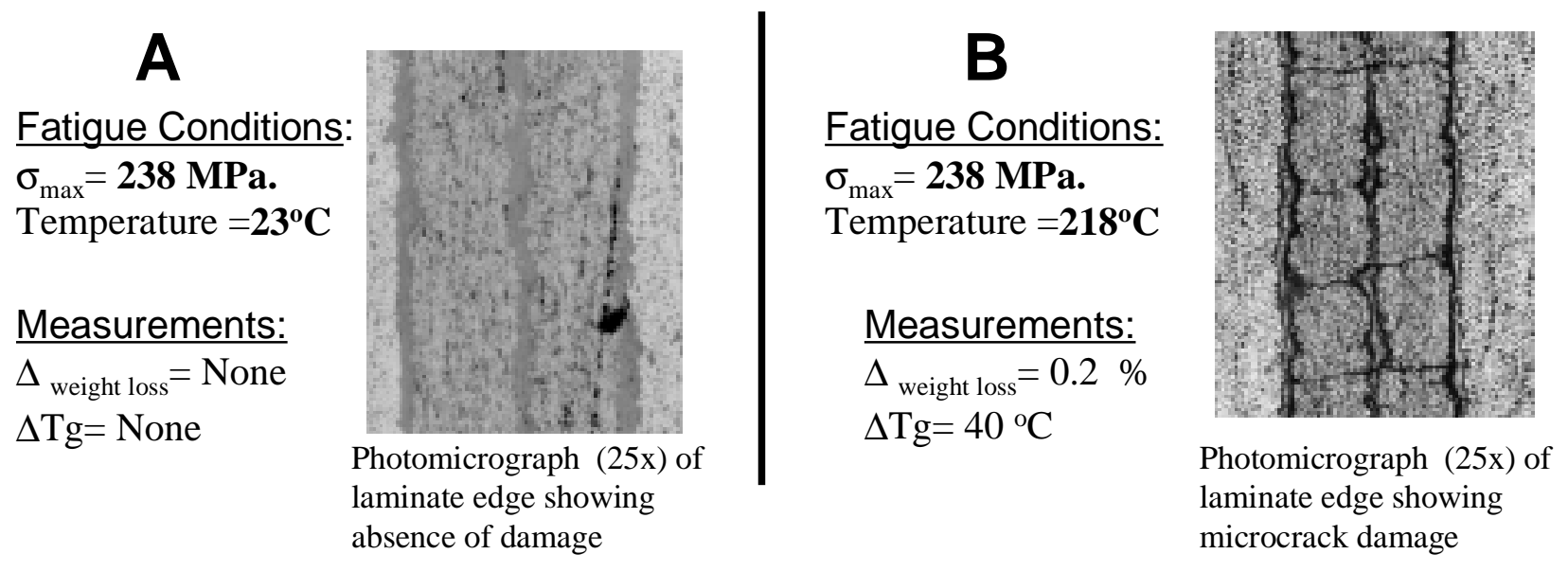

Figure 2. Effects of temperature during isothermal fatigue on the residual weight, glass transition, and damage state of a graphite/bismaleimide composite. 

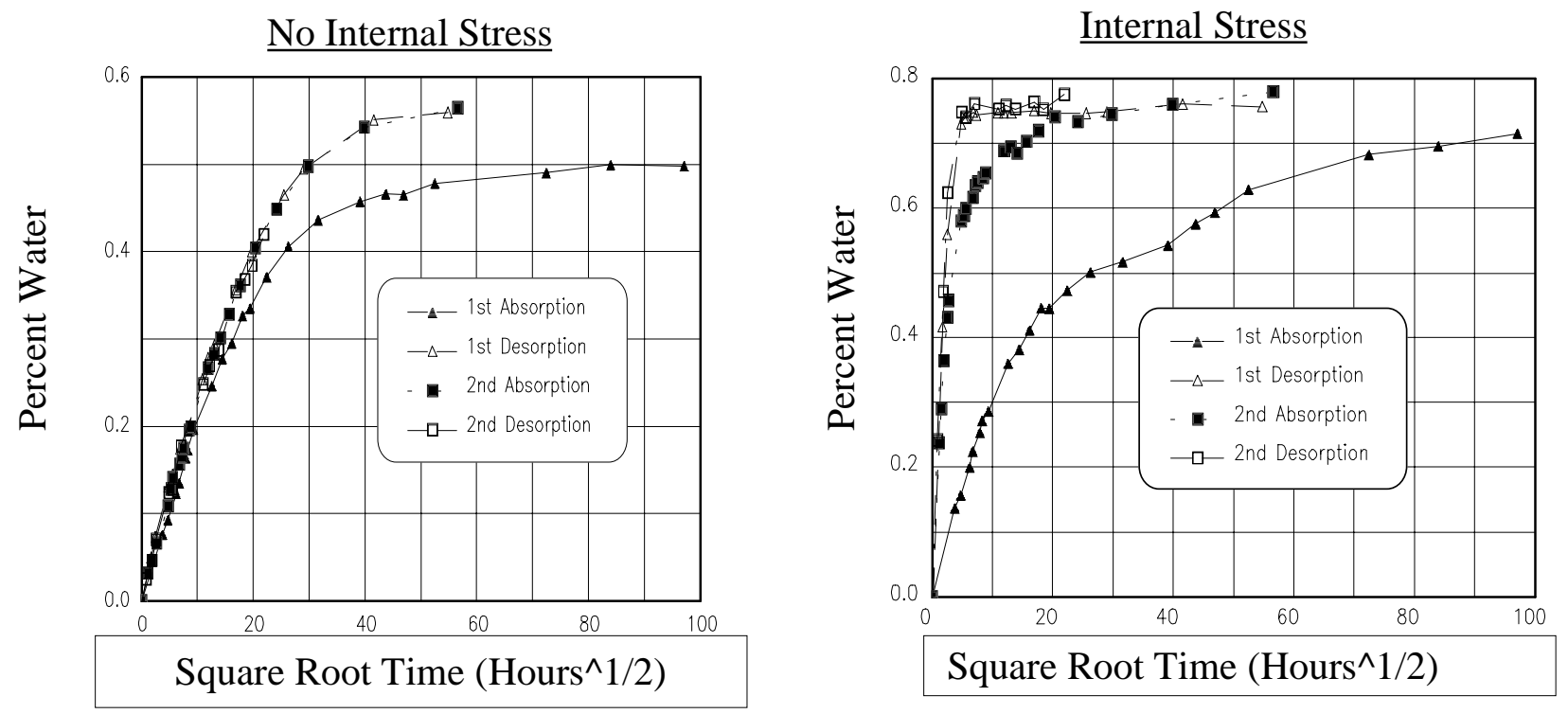

Figure 3. Effects of internal stress on the measured water uptake of a graphite/thermoplastic.

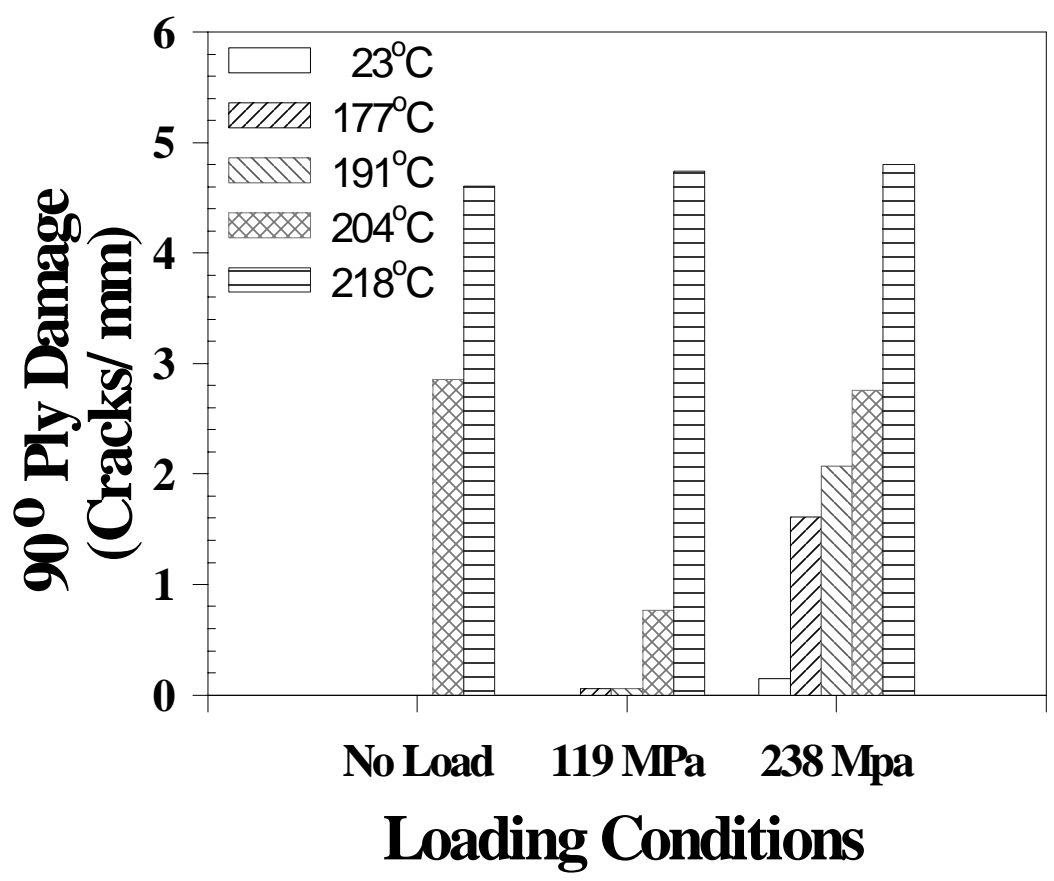

Figure 4. Effects of test temperature and fatigue stress level on the development of matrix cracks of a graphite/bismaleimide composite. 